\title{
High Speed Electrical Discharge Machining Using 3-DOF Controlled Magnetic Drive Actuator
}

\author{
Xiaoyou Zhang ${ }^{*}$, Satoshi Tanaka \\ Department of Mechanical Engineering, Nippon Institute of Technology, Japan
}

Copyright (C) 2015 by authors, all rights reserved. Authors agree that this article remains permanently open access under the terms of the Creative Commons Attribution License 4.0 International License

\begin{abstract}
Electrical discharge machining (EDM) has several advantages, such as the capability of machining all conductive materials regardless of their hardness and the ability to deal with complex shapes. In EDM, the machining speed largely depends on the probability and efficiency of the electrical discharges. To obtain a stable electrical discharge, it is necessary to maintain a suitable gap between the electrode and the workpiece. In this study, a three-degree-of-freedom (3-DOF) controlled, wide-bandwidth, high-precision, long-stroke magnetic drive actuator was developed to allow the rapid positioning of the electrode, and EDM experiments were conducted using the developed actuator to confirm the increase in the machining speed. Furthermore, planetary machining experiments were also performed to determine if the developed actuator can be used to adjust the diameter of the machined holes. The experimental results demonstrate that the actuator can rapidly adjust the gap between the electrode and the workpiece. In the experiments, using the actuator increased the machining speed by $89 \%$ in comparison with using only the conventional electrical discharge machine. In addition, the diameter of the machined hole can be adjusted by using the multi-DOF positioning function of the actuator.
\end{abstract}

Keywords Electrical Discharge Machining, Actuator, Magnet, High Speed

\section{Introduction}

Electrical discharge machining (EDM) is an unconventional material removal process based on the thermoelectric energy created between an electrically conductive workpiece and an electrode submerged in a machining fluid [1]. EDM has several advantages, such as the capability of machining all conductive materials regardless of their hardness and the ability to deal with complex shapes [2][3]. Therefore, EDM has been traditionally used to machine complex molds and has also been applied to the machining of micro-holes in fuel injection and ink-jet nozzles.

In a normal electrical discharge machine, a combination of stacked one-degree-of-freedom (1-DOF) lead screw mechanisms is typically used to position the electrode in three orthogonal directions [4]. However, the positioning response of a lead screw is somewhat slow because of the mass of the stacked tables and the rotational inertia of the lead screws, and thus the speed and accuracy of conventional EDM are limited by the probability and efficiency of electrical discharges [5]. To improve the machining speed and accuracy, a combination of a conventional electrical discharge machine and a wide-bandwidth, high-precision local actuator is necessary.

A piezoelectric actuator and a voice coil motor, which improves the positioning response of electrodes, have been developed as additional local actuators by Higuchi et al. [6] and Masuzawa et al. [7], respectively. Using these actuators, the electrode can be positioned at a high speed. However, the DOFs of the motion are restricted to one direction. Zhang et al. [8][9] developed a maglev local actuator to position the electrode in 5 DOFs. The actuator is compact enough to be attached to conventional electrical discharge machines, and it has a stroke of a few millimeters in the thrust direction, realizing the jump operation of the electrode. However, in the radial direction, the actuator has a stroke of only $200 \mu \mathrm{m}$, which is not large enough to realize three-dimensional (3D) surface EDM.

In this study, a 3-DOF controlled, wide-bandwidth, high-precision, millimeter-stroke magnetic drive actuator was developed and attached to an electrical discharge machine to experimentally confirm that it can increase the machining speed. The possibility of adjusting the diameters of machined holes using the 3-DOF positioning function of the actuator was also examined. 


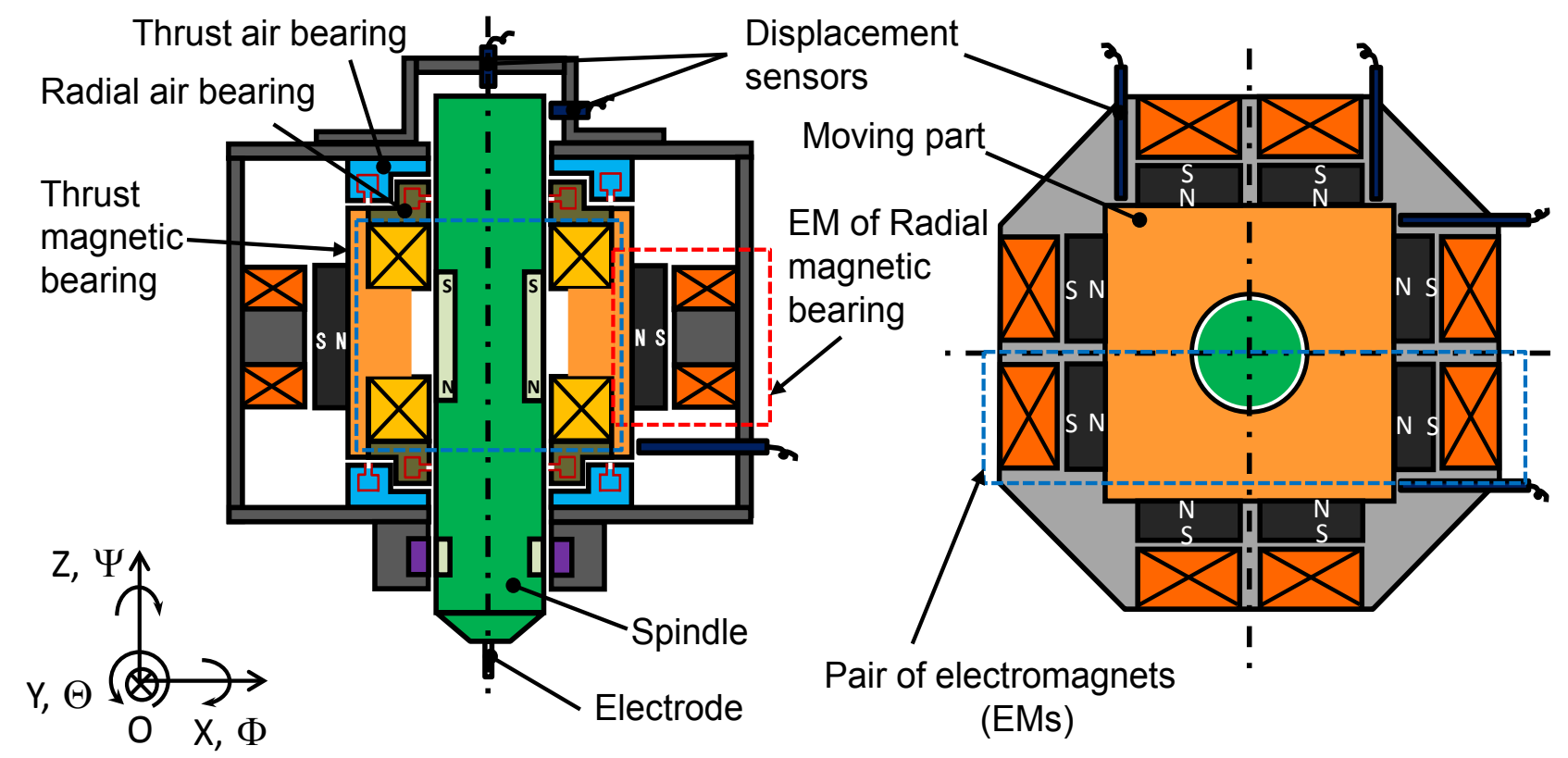

Figure 1. Configuration of 3-DOF controlled magnetic drive actuator

\section{Magnetic Drive Actuator}

\subsection{Magnetic Drive Actuator Mechanism}

Fig. 1 shows the mechanism of the proposed 3-DOF controlled magnetic drive actuator. The actuator primarily consists of thrust and radial magnetic bearings and thrust and radial air bearings. Using the thrust magnetic bearing, the motion of the spindle attached to an electrode in the $\mathrm{Z}$ direction can be controlled. The motions of the spindle in the $\mathrm{X}, \mathrm{Y}, \Theta$ and $\Phi$ directions are constrained by the upper and lower radial air bearings.

The radial magnetic bearing consists of four pairs of electromagnets (EMs): two oriented in the $\mathrm{X}$ direction and two in the $\mathrm{Y}$ direction. In each pair, the two EMs are on opposite sides of the moving part. In the $\mathrm{X}$ or $\mathrm{Y}$ direction, if the electromagnetic force of one pair of EMs is in the same direction as another pair, the translational motion of the moving part and the spindle is controlled. In contrast, if the electromagnetic forces of two pairs of EMs are in opposite directions, the rotational motion of the moving part about the $\mathrm{Z}$-axis is constrained. Moreover, the motion of the moving part in the $Z, \Theta$ and $\Phi$ directions is constrained by the upper and lower thrust air bearings. Therefore, the thrust and radial magnetic bearings can be used to control the motions of the spindle in the $\mathrm{X}, \mathrm{Y}$ and $\mathrm{Z}$ directions.

\subsection{Experimental Magnetic Drive Actuator and Its Performance}

Fig. 2 shows a photograph of the developed experimental magnetic drive actuator and its spindle. The height, width, and mass of the actuator are $125 \mathrm{~mm}, 172 \mathrm{~mm}$, and $9.5 \mathrm{~kg}$, respectively. The length, diameter, and mass of the spindle without its attachments are $121 \mathrm{~mm}, 20 \mathrm{~mm}$, and $0.27 \mathrm{~kg}$, respectively. The air-core coils of the thrust and radial magnetic bearings have 208 and 588 turns, respectively, and the permanent magnets are of $\mathrm{Nd}_{2} \mathrm{Fe}_{14} \mathrm{~B}$.
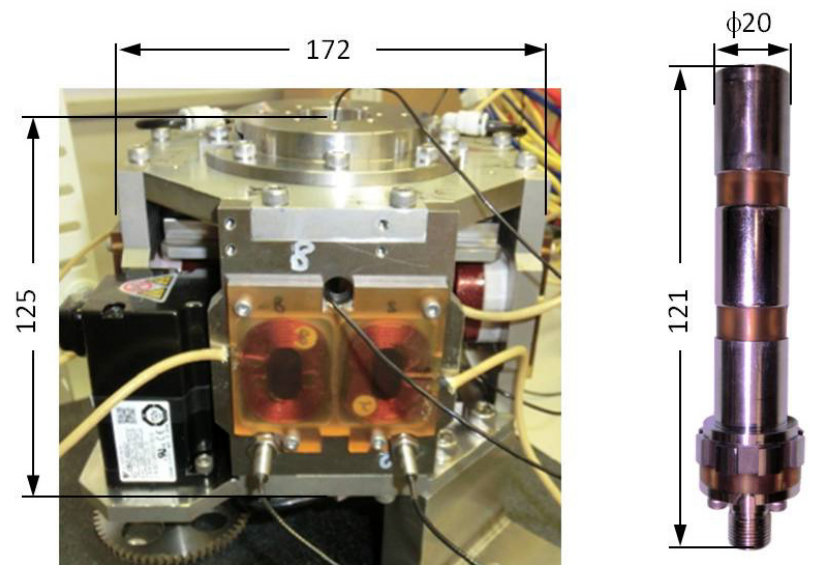

Figure 2. Experimental magnetic actuator and its spindle

The displacements of the spindle in the thrust and radial directions are measured by three eddy current displacement sensors (PU-05, AEC Corp., measurement range $\pm 1.0 \mathrm{~mm}$, resolution $0.5 \mu \mathrm{m}$ ), and the displacements of the moving part in the radial direction are measured by the same type of sensor (PU-05). The actuator is controlled via a digital signal processor (DSP; DS1103 PPC Controller Board, dSPACE Corp.) with a sampling rate of $10 \mathrm{kHz}$. The actuator has a positioning resolution of $0.75 \mu \mathrm{m}$ and a positioning stroke of $2 \mathrm{~mm}$ in the directions of the 3 DOFs. The bandwidth is 110 $\mathrm{Hz}$ in the $\mathrm{Z}$ direction and $50 \mathrm{~Hz}$ in the $\mathrm{X}$ and $\mathrm{Y}$ directions. 


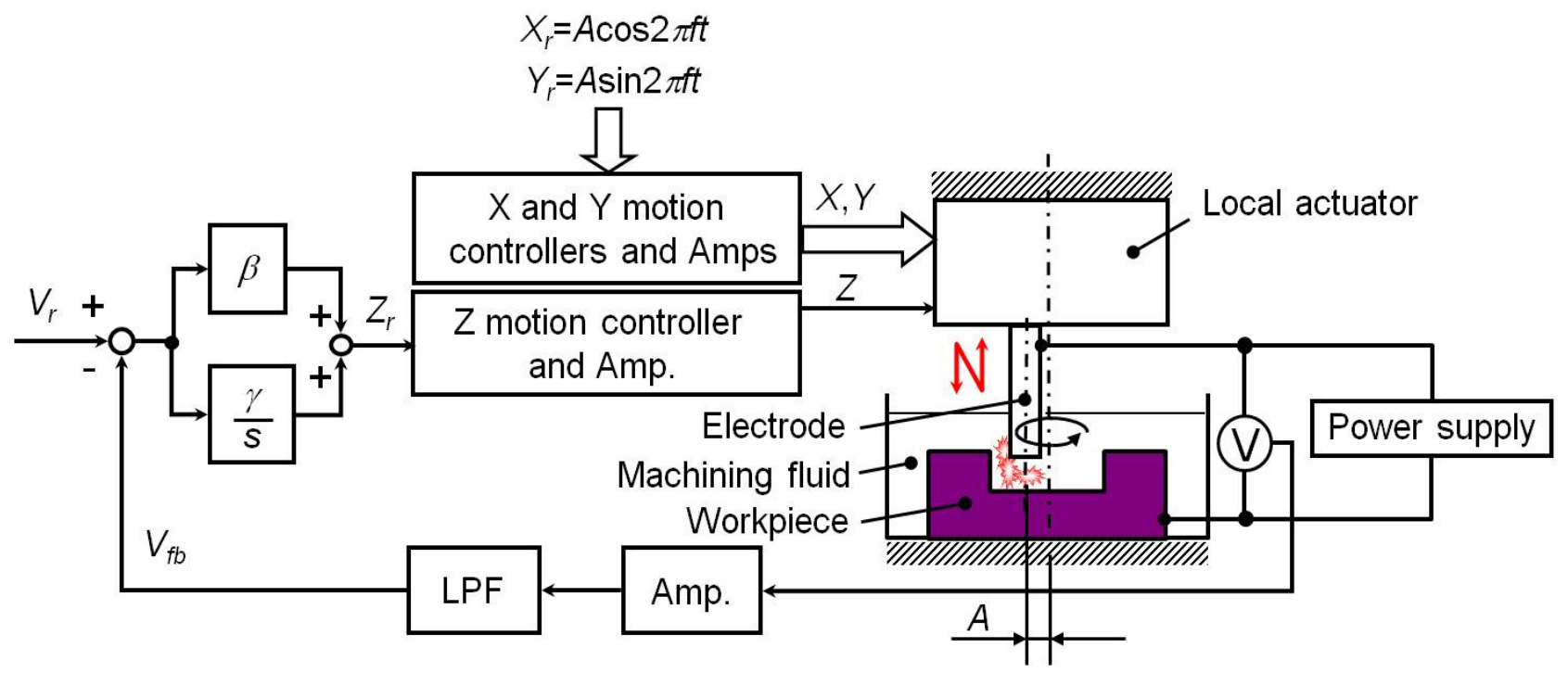

Figure 3. Block diagram of EDM system

\section{Electrical Discharge Machining System Using the Local Actuator}

To verify the effect of the local actuator on the EDM stability and speed and examine the possibility of adjusting the diameters of machined holes, the developed actuator was attached to an electrical discharge machine (EA8PV, Mitsubishi Electric Corp.). In the EDM experiments, the conventional electrical discharge machine was used only to move the electrode to the initial position and supply a voltage to the electrode. The electrode was controlled solely by the local actuator.

A cylindrical copper electrode was used which was not rotated or retracted during EDM. The machining fluid was EDF-K (Nippon Oil Corp.). The EDM experiments were conducted until a 0.5 -mm-thick SUS304 steel plate was penetrated. The discharge circuit was a transistor circuit with a peak current of 9.0 A and pulse on- and off-times of 44.8 and $57.6 \mu \mathrm{s}$, respectively.

To maintain a stable electrical discharge, the voltage between the electrode and the workpiece was kept at a constant value by rapidly changing the distance between the electrode and the workpiece. A block diagram of the EDM system is shown in Fig. 3. In this system, the gap voltage $V$ is amplified and averaged by a low-pass filter (LPF), and the average voltage $V_{f b}$ is used as a feedback signal. The gain of the amplifier is 0.03 , and the cutoff frequency of the low-pass filter is $330 \mathrm{~Hz}$. The deviation between the reference voltage $V_{r}$ and the feedback voltage $V_{f b}$ is sent to a PI controller to calculate $V_{a r}$, which is used to position the electrode with respect to the workpiece. The references $\mathrm{Xr}$ and $Y r$ describe the path of the electrode. To produce a stable electrical discharge, the reference voltage $V_{r}$ was set to 1.66 $\mathrm{V}$, and the values of $\beta$ and $\gamma$ in the PI controller were set to $1.0 \times 10^{-5}$ and $1.0 \times 10^{-4}$, respectively; these values were selected by trial and error.

\section{Electrical Discharge Machining Experiments}

\subsection{Hole Machining}

To evaluate how effectively the proposed actuator improves the EDM machining speed, straight holes were machined in the thrust direction. In the EDM experiments, a copper electrode with a diameter of $1 \mathrm{~mm}$ was used, and the electrode position in the thrust direction was controlled by the voltage across the gap between the electrode and the workpiece. For comparison, an EDM experiment was also conducted using only the conventional electrical discharge machine. The parameters of the PI controller and the machining conditions were identical to those used for the actuator.

Fig. 4(a) and (b) shows photographs of the machined holes without and with the proposed actuator, respectively. The diameters of the holes machined using the proposed actuator and using only the conventional electrical discharge machine were 1.068 and $1.064 \mathrm{~mm}$, respectively; these two diameters were almost the same. The feed displacements of the electrode in the thrust direction were measured to evaluate the motion of the electrode during EDM, as shown in Fig. 5. By using the proposed actuator, the machining time was reduced from 292.9 to $167.2 \mathrm{~s}$.

Moreover, the feedback voltage $V_{f b}$ was monitored by a digital signal processor (DS1103) with a sampling rate of 10 kHz., as shown in Fig. 6. Comparing Fig. 6(a) with Fig. 6(b), it can be seen that the response of the electrode to maintain a stable electrical discharge is enhanced when using the proposed actuator. 


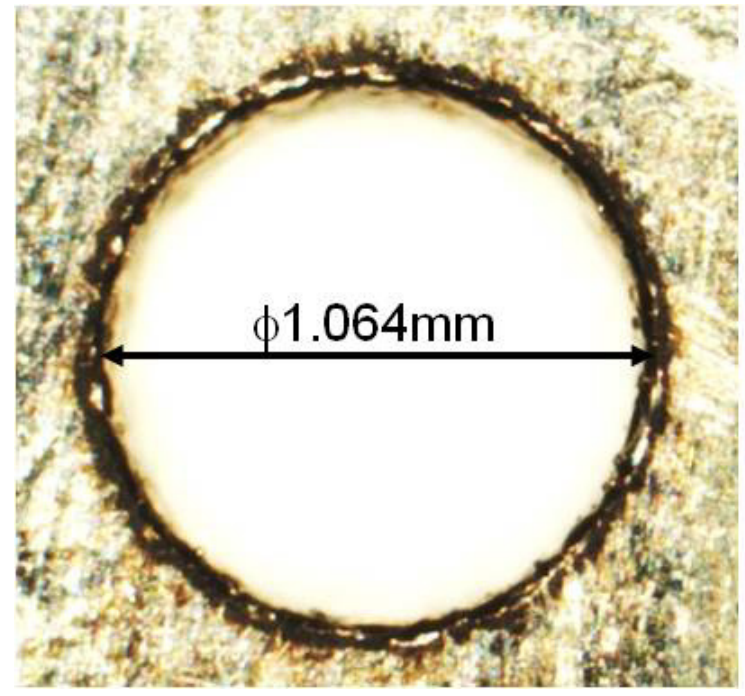

(a) Conventional electrical discharge machine

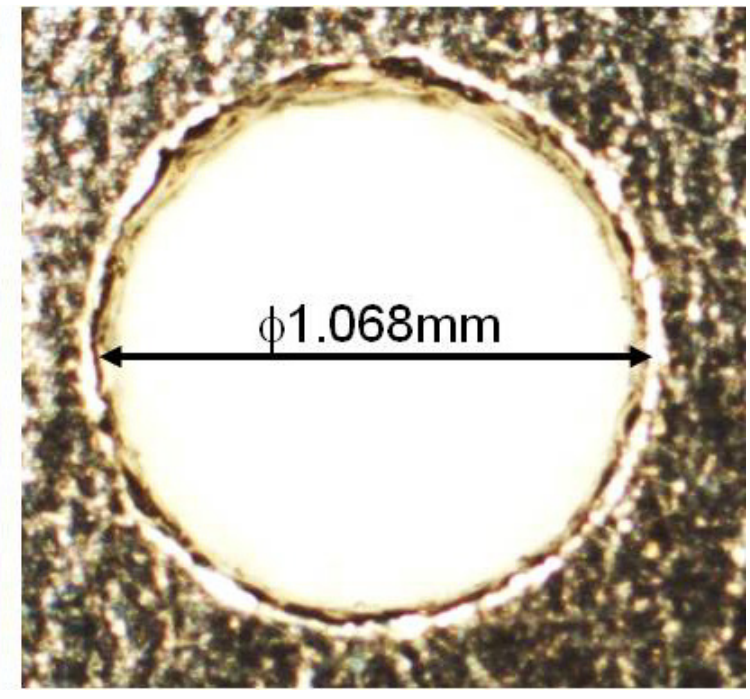

b) Actuator

Figure 4. Machined holes

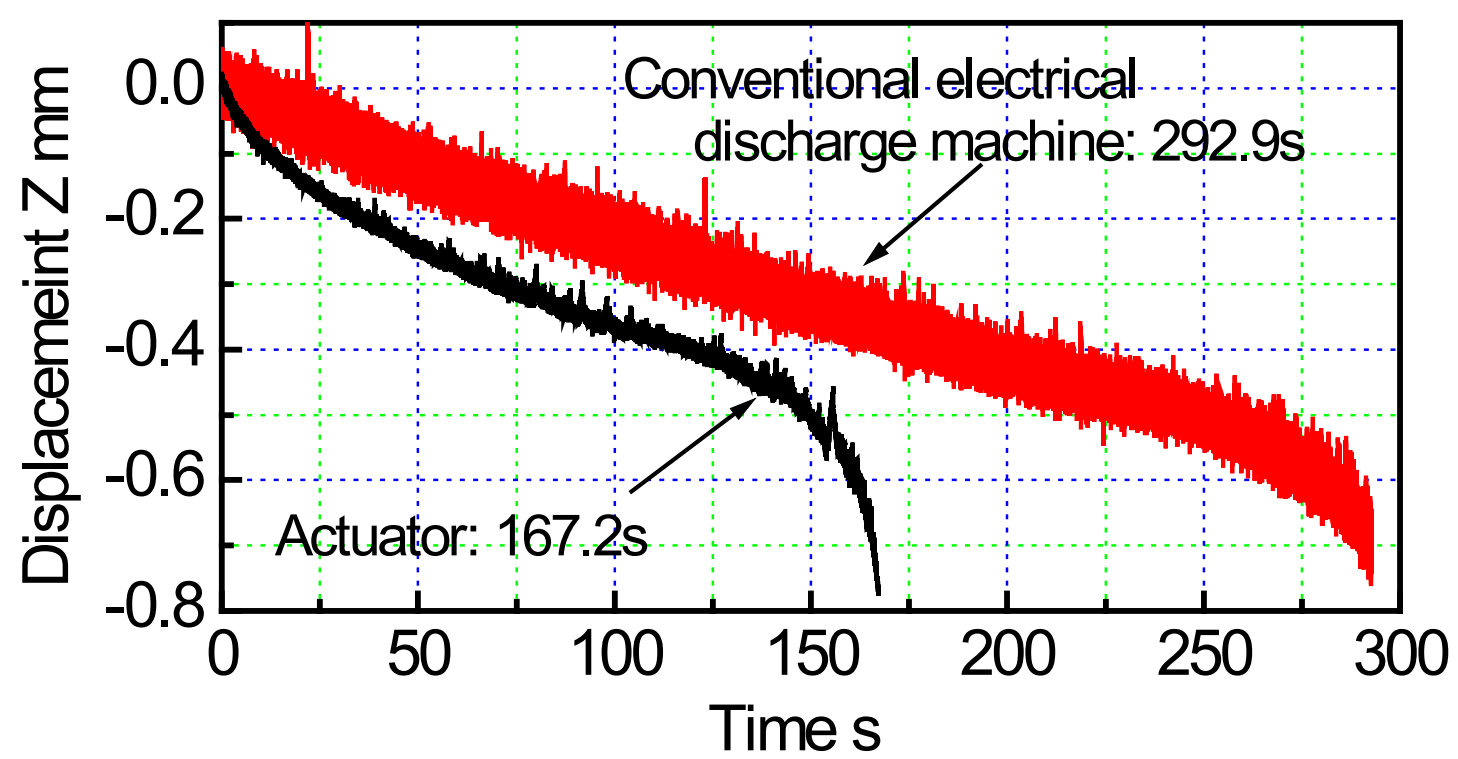

Figure 5. Feed displacements of electrode
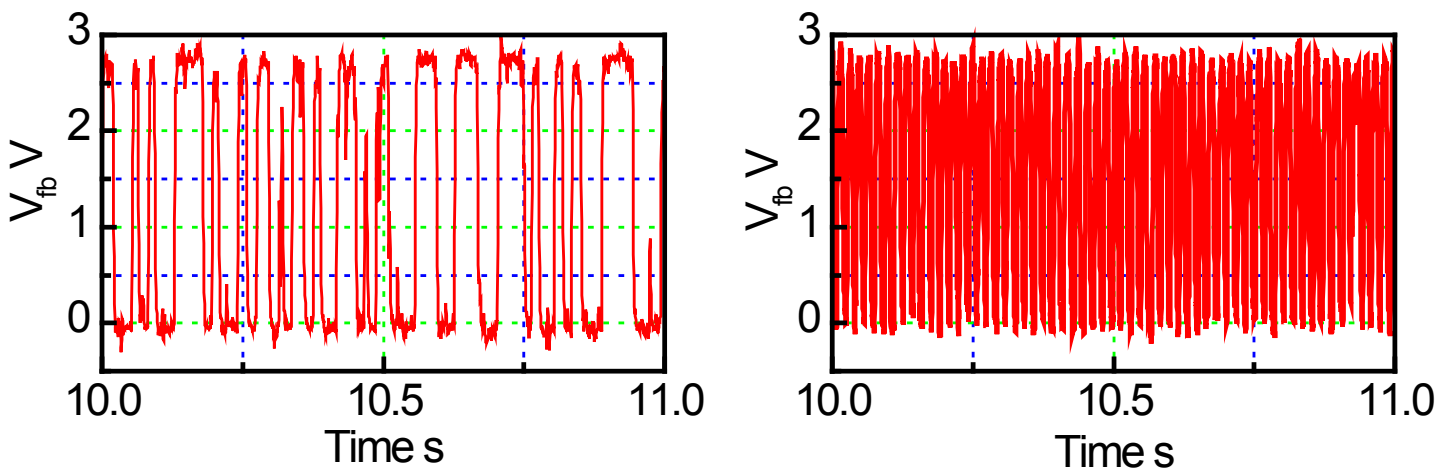

Figure 6. Feedback voltage $V_{f b}$ during EDM

For each EDM experiment, several holes were machined under the same conditions. Ten holes were used for the sample data, excluding the holes influenced by large abnormal electrical discharges. The calculated average machining speed, which 
is defined as the ratio of the thickness of the workpiece to the average machining time, is shown in Fig. 7. When machining was conducted using the local actuator and using only a conventional electrical discharge machine, the average machining speeds were 2.99 and $1.61 \mu \mathrm{m} / \mathrm{s}$, respectively. Thus, the actuator improved the average machining speed by $86 \%$ in comparison with using only a conventional electrical discharge machine.

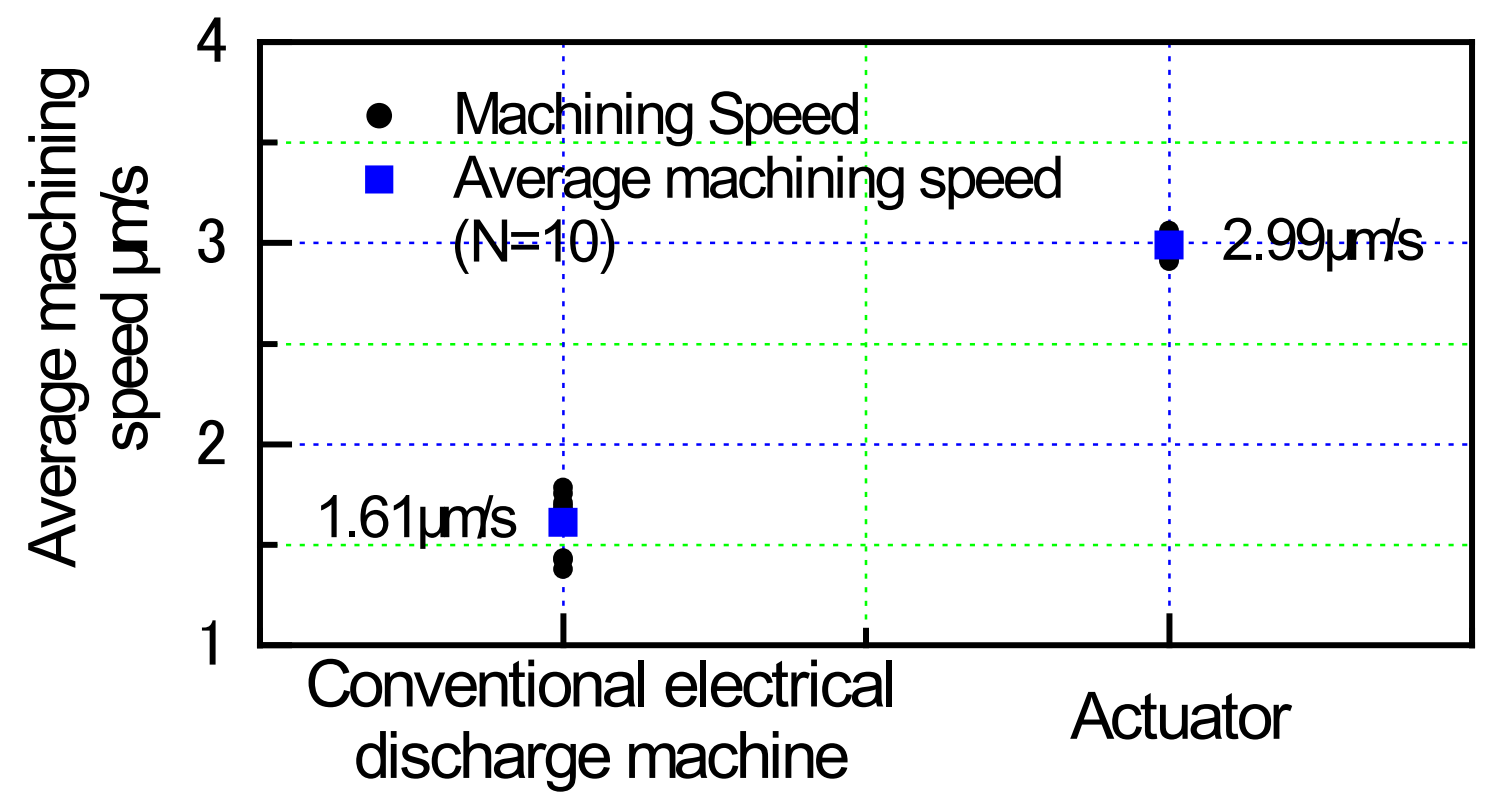

Figure 7. Average machining speed

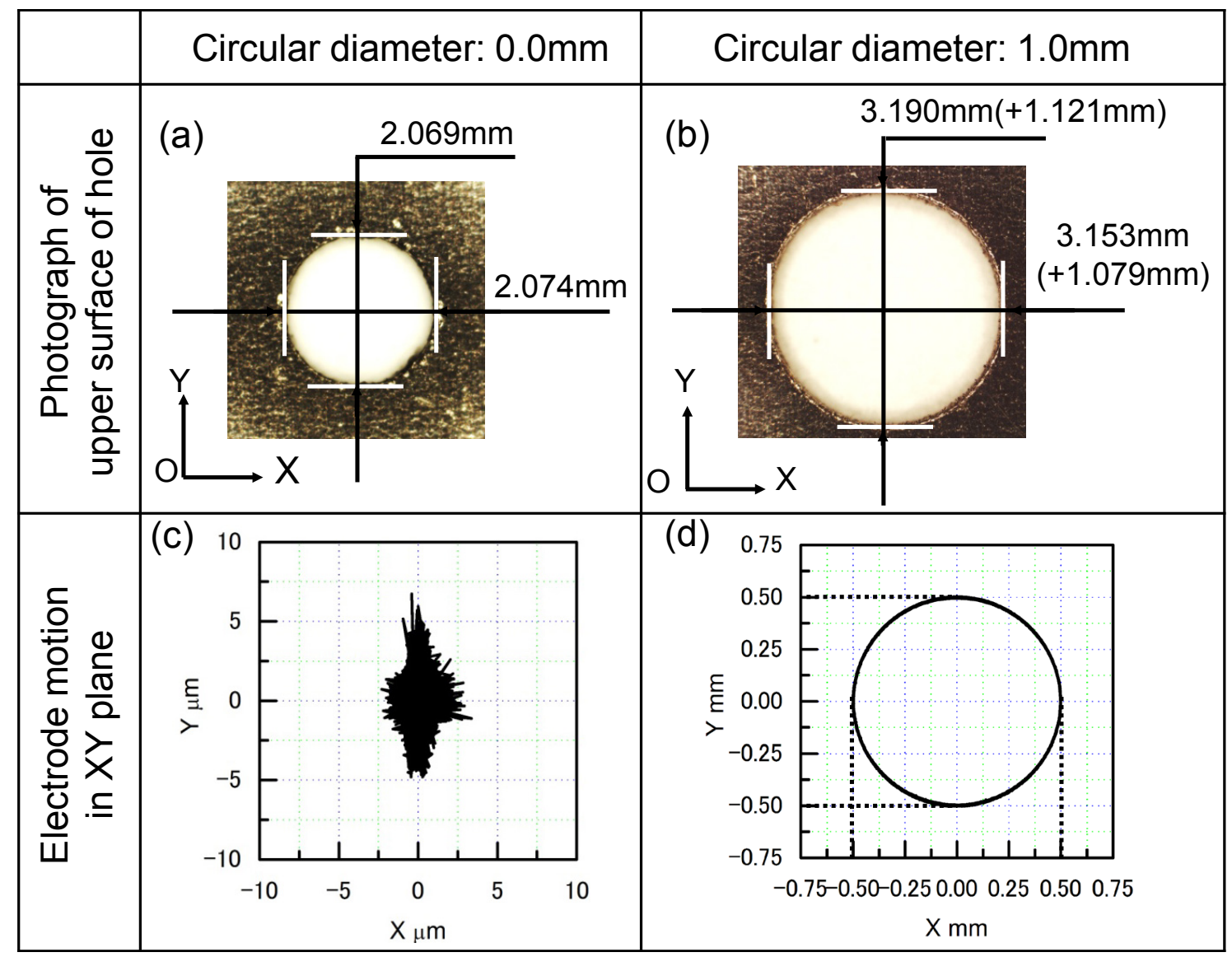

Figure 8. Planetary machining 


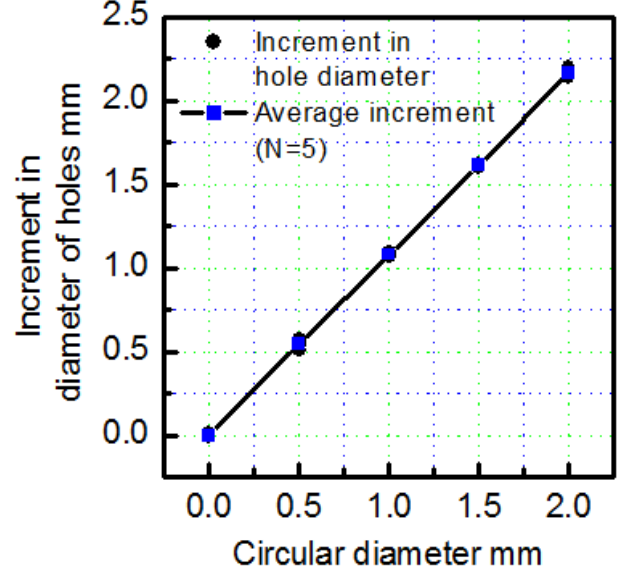

(a) $\mathrm{X}$ direction

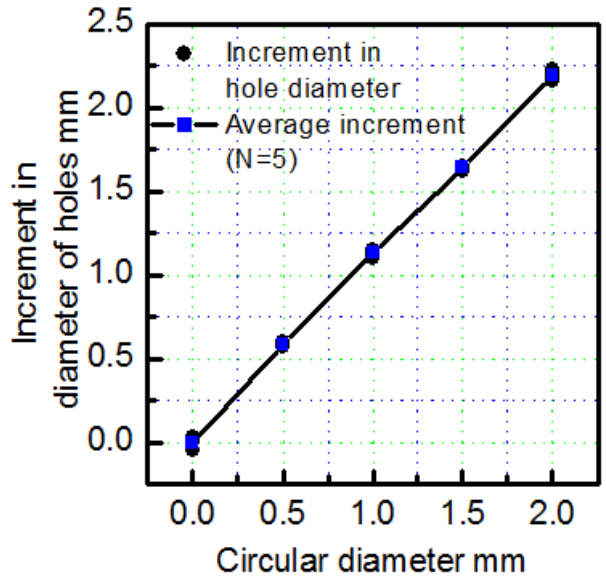

(b) Y direction

Figure 9. Relationship between the increment in the hole diameter and the diameter of the circular path of the electrode

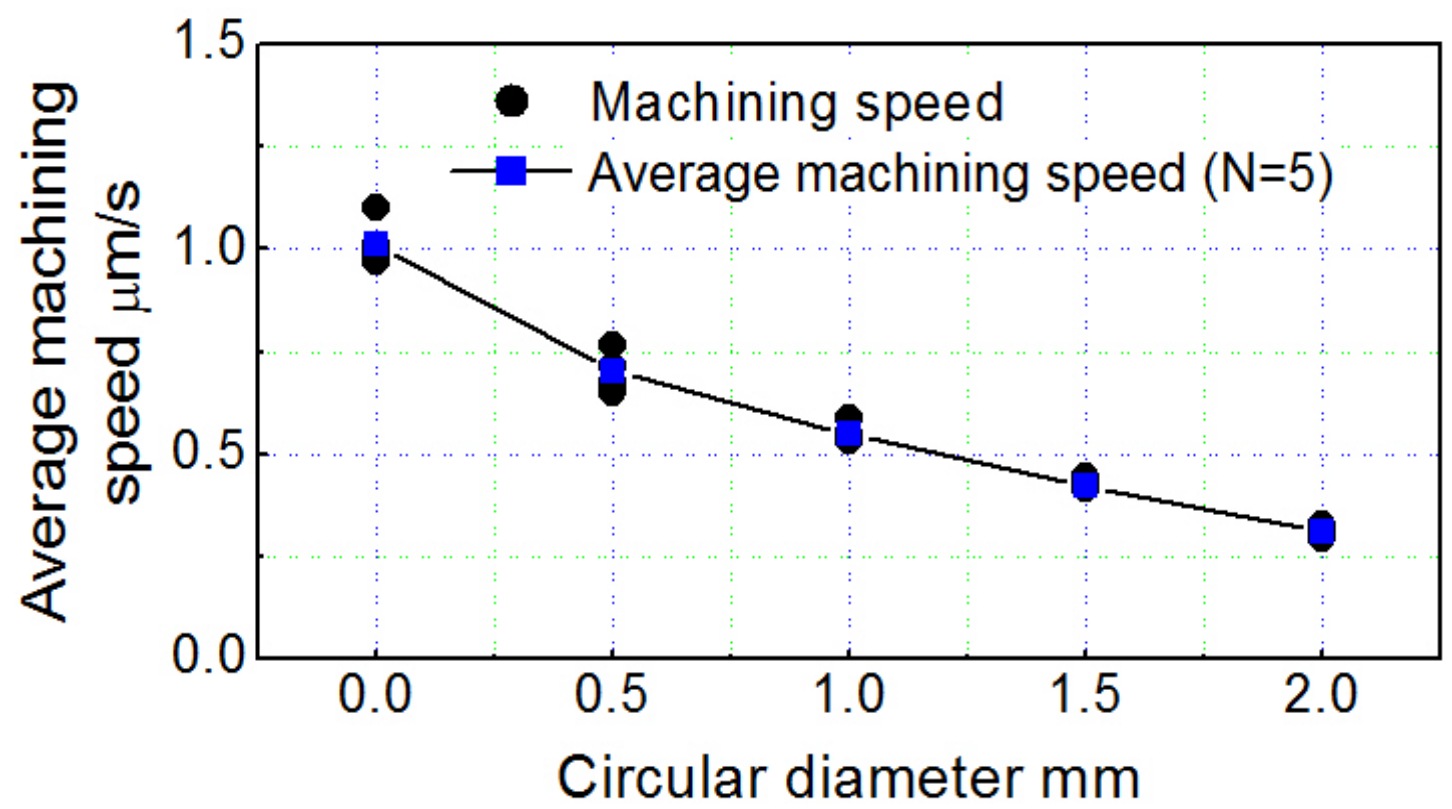

Figure 10. Average machining speed in planetary machining

\subsection{Planetary Machining}

The actuator can be used not only to machine holes but also to adjust their diameters. This is done by positioning the electrode such that the gap voltage is constant in the $\mathrm{Z}$ direction and driving the electrode in a circular path in the $\mathrm{XY}$ plane. This technique is called planetary machining.

For the circular path, the reference values for the motion in the $\mathrm{X}$ and $\mathrm{Y}$ directions are given as follows:

$$
\left\{\begin{array}{c}
X_{r}=A \cos 2 \pi f t \\
Y_{r}=A \sin 2 \pi f t
\end{array}\right.
$$

where $A$ and $f$ are the radius and frequency of the motion, respectively. In this experiment, the radius was set to 0.25 , $0.5,0.75$, and $1.0 \mathrm{~mm}$, the frequency was set to $10 \mathrm{~Hz}$, and an electrode with a diameter of $2 \mathrm{~mm}$ was used.

The upper surfaces of the machined holes and the paths of the electrode in the XY plane are shown in Fig. 8. When the diameter of the circular path was set to $0 \mathrm{~mm}$, the hole spanned 2.074 and $2.069 \mathrm{~mm}$ in the $\mathrm{X}$ and $\mathrm{Y}$ directions, respectively. When the diameter of the circular path was increased to $1.0 \mathrm{~mm}$, the hole spanned 3.153 and $3.190 \mathrm{~mm}$ in the $\mathrm{X}$ and $\mathrm{Y}$ directions, respectively. Thus, the diameter of the hole was expanded by approximately $1 \mathrm{~mm}$, which is equal to the diameter of the circular path.

To investigate the effect of increasing the diameter of the circular path on the increment in the hole diameter, five holes were machined under the same conditions, and the diameter of the circular path was increased from 0.0 to $2.0 \mathrm{~mm}$ in increments of $0.5 \mathrm{~mm}$. Fig. 9 shows the relationship between the increment in the diameters of the five holes and the 
diameter of the circular path. The experimental results demonstrate that the diameter of the machined hole changes in proportion to the diameter of the circular path.

The average machining speeds for circular paths with different diameters are shown in Fig. 10. As the diameter of the circular path increased, the average machining speed of the holes decreased. This may be because the electrical discharge in planetary machining becomes unstable in the $\mathrm{Z}$ direction as the diameter of the circular path increases. Thus, for planetary machining, retraction limited to the $\mathrm{Z}$ direction is insufficient to avoid short circuits in the radial and thrust directions.

\section{Summary}

In this study, a 3-DOF controlled, wide-bandwidth high-precision, long-stroke magnetic drive actuator was developed to maintain a suitable gap between the electrode and the workpiece. The actuator has a positioning resolution of $0.75 \mu \mathrm{m}$ and a positioning stroke of $2 \mathrm{~mm}$ in the directions of the 3 DOFs. The bandwidth is $110 \mathrm{~Hz}$ in the $\mathrm{Z}$ direction and $50 \mathrm{~Hz}$ in the $\mathrm{X}$ and $\mathrm{Y}$ directions.

To evaluate how effectively the proposed actuator improves the EDM speed, straight holes were machined in the thrust direction using the proposed actuator. The experimental results demonstrate that the proposed actuator can rapidly adjust the distance between the electrode and the workpiece. Additionally, when using the proposed actuator, the machining speed increased by $86 \%$ in comparison with using only the conventional electrical discharge machine. Moreover, by employing the planetary motion of the electrode, the diameter of the machined hole can be increased by up to approximately $2 \mathrm{~mm}$. In future work, we plan to investigate the machining of 3D microstructures.

\section{Acknowledgements}

This study was supported in part by JSPS KAKENHI
Grant Number 23560169 and NSK Foundation for the Advancement of Mechatronics.

\section{REFERENCES}

[1] Kunieda, M., Lauwers, B., Rajurkar K. P., \& Schumacher, B. M. (2005), Advancing EDM through Fundamental Insight into the Process. Annals of the CIRP, vol.54, no.2, pp. 599-622.

[2] Ho, H., \&Newman, S. T. (2003), State of the Art Electrical Discharge Machining (EDM). International Journal of Machine Tools and Manufacture, vol. 43, no. 13, pp. $1287-1300$.

[3] Masuzawa, T. (2000). State of Art of Micromachining. Annals of the CIRP, vol.49, no. 2, pp. 473-488.

[4] http://www.diax-net.com/

[5] Varanasi, K. K., Nayfeh, S. A. (2004). The dynamics of lead-screw drives: Low-Order modeling and experiments. Trans ASME J Dyn Syst Meas Control, vol. 126, pp. 388-396.

[6] Higuchi T., Furutani K., Yamagata Y., Takeda K. (1991). Development of pocket-size electro-discharge machine. Ann CIRP, vol. 40, no. 1, pp. 203-206.

[7] Masuzawa T., Tanaka K., Fujino M. (1978). Study on the high speed machining by EDM using a moving coil head type feed control. Proceedings of the 19th International MTDR Conference. pp. 543-549.

[8] Zhang, X., Shinshi, T., Kajiwara, G., Shimokohbe, A., Imai, Y., Miyake, H., \& Nakagawa, T. (2008). A 5-DOF Controlled Maglev Local Actuator and its Application to Electrical Discharge Machining, Precision Engineering, vol. 32 , no. 4 , pp. 289-300.

[9] Zhang, X., Shinshi, T., Shimokohbe, A., Soto, T., Miyake, H., \& Nakagawa, T. (2008). High-speed Electrical Discharge Machining By Using a 5-DOF Controlled Maglev Local Actuator, Journal of Advanced Mechanical Design, Systems, and Manufacturing, vol. 2, no. 4, pp. 493-503. 